\title{
Evaluation of Selective Broadcast Algorithms for Safety Applications in Vehicular Ad Hoc Networks
}

\author{
Kaan Bür and Maria Kihl \\ Department of Electrical and Information Technology, Lund University, P.O. Box 118, 22100 Lund, Sweden \\ Correspondence should be addressed to Kaan Bür, kaan.bur@eit.lth.se
}

Received 15 September 2010; Revised 17 December 2010; Accepted 8 February 2011

Academic Editor: Syed R. Rizvi

Copyright $\odot 2011$ K. Bür and M. Kihl. This is an open access article distributed under the Creative Commons Attribution License, which permits unrestricted use, distribution, and reproduction in any medium, provided the original work is properly cited.

Just as wireless communications develop further to achieve higher performance, new application areas emerge to challenge the limits. Vehicular ad hoc networks are one of these areas, and emergency situation warning is one of their most popular applications since traffic safety is a concern for everyone. Due to the life-critical nature of emergency applications, however, it is extremely important to ensure the solutions proposed meet the standards required, such as reliable and timely delivery of the safety warning in a situation like car collision avoidance. In order to put the candidate solutions to the test and evaluate their feasibility, we adopt the approach of computer simulation. We implement four different selective broadcast algorithms used for information dissemination in vehicular ad hoc networks, and compare their performance under identical realistic simulation conditions. Our goal is to provide an evaluation focussing on the performance with respect to safety, rather than to network aspects like throughput, loss, and delay. We define four new performance criteria to address the effectiveness, efficiency, timeliness, and overhead of the broadcast algorithms in safety warning delivery. The results we obtain using these criteria help us to understand better the design requirements of a high-performance selective broadcast algorithm.

\section{Introduction}

In light of the average speeds and car following distances observed on today's highways, the drivers' reactions to unexpected road hazards are dangerously slow. Driver perceptionresponse time, defined as the time from the first sighting of an obstacle on the road until the application of the brakes, is measured in various experiments, and the results presented in [1] indicate a reaction time of about $1.6 \mathrm{~s}$ for $95 \%$ of the human subjects. As shown in [2], this is not a time long enough to avoid collisions in many emergency cases, especially when the driver violates the safety distance rule or the road and weather conditions limit the ability of the driver to spot the emergency event from a distance. Under these conditions, a collision avoidance strategy based solely on the tail brake lights of the cars ahead has a high probability of failure. What we need is an early warning system to inform the drivers on the emergency situation arising ahead of the road but still outside the driver's vision, triggering the driver's reaction well in advance so collision avoidance is possible even at a perception-response time as high as $1.6 \mathrm{~s}$.
The developments in electronics as well as telecommunications, which led to handheld computers with mobile communication and environmental sensing capabilities, have also had a profound effect on the automobile industry. As discussed in [3], modern vehicles are becoming increasingly intelligent, already equipped with effective driver assistance and passive safety functions. The development of more complex, active safety functionality, on the other hand, requires accurate positioning and classification of objects around the vehicle. Today, this data is provided by invehicle sensors like radar and camera. However, in-vehicle sensors cannot detect out-of-sight objects around corners or behind summits, position objects accurately at large range, or detect all attributes like weight, tire-road friction, and intended direction. This information needs to be made available through a communication link. With the assistance of vehicular communication systems, active traffic safety applications like collision avoidance and notification can be developed, which can, in return, lower considerably the accident rates. 
There are mainly two realistic ways to form a vehicular network [4-6]. First, the vehicles can organise themselves to form an ad hoc network, that is, a spontaneous, mobile network to operate without the help of an infrastructure and transmit data in a single- or multihop fashion. Roadside units can be included in the ad hoc network, providing the vehicles with Internet access and centralised services. Second, infrastructure-based communication technologies can be used, where vehicles transmit data to each other via a roadside base station. The vehicles do not need to be aware of each other, since the base stations have total control of the network. In this paper, we focus on the former alternative, known as vehicular ad hoc networks (VANET), where all communication is performed without any infrastructure.

Safety applications have stringent real-time operation and reliability requirements, typically represented by message frequency, delay, and loss [3]. Considering the missioncritical nature of the traffic safety applications, it is of extreme importance that, once the emergency situation arises, the warning reaches in a timely and reliable manner as many of the vehicles as possible, which are potentially endangered by the situation. Given the variable density of the vehicular network, the high speed of as well as big speed differences between its nodes, the possible coexistence of many wireless applications sharing the available bandwidth, and the unstable wireless channel conditions the network must operate under, the reliability and timeliness of the traffic safety application depends mainly on two factors at the network level: the method it uses for data dissemination and the network overhead it generates. An efficient data dissemination strategy has to ensure a high success rate regarding the timely delivery of the emergency message, while maintaining a low resource usage profile. This also reduces the probability of contention as well as packet loss in the network, yielding lower delays and, thus, helping the network to cope with the application's quality of service (QoS) requirements more easily.

The key to the successful dissemination of safety warnings is the decision mechanism employed for message delivery and repetition, that is, finding an intelligent way of deciding when and how a safety message should be delivered or repeated to maximise reliability while keeping the overhead low. Given the properties of safety applications and the limitations of vehicular communications, selective broadcast or multicast strategies (topology versus location based, reactive approaches) seem more applicable than both unicast routing (introducing more complexity and less redundancy) as well as flooding (generating a high overhead without increasing the success rate substantially) $[4,6,7]$. Several solution proposals have been made to introduce intelligence to the basic broadcast concept, in order to make it more selective and, thus, more efficient in its resource usage, while not jeopardising the high warning delivery rate required by the traffic safety application. Various selective broadcast algorithms have been developed as part of these proposals [2], which require careful examination under realistic test scenarios. Moreover, it is also necessary to compare these different algorithms within the framework of a unified methodology, that is, in a single environment and under the same conditions. In this paper, we present such an evaluation by way of simulation.

The rest of this paper is organised as follows. In Section 2, we summarise a selection of selective broadcast schemes and some of the research efforts directed at their evaluation. Section 3 presents our evaluation scenario, starting with a description of the algorithms under investigation, followed by the explanation of the simulation settings we chose for a realistic evaluation. In Section 4, we introduce our performance criteria and compare the algorithms according to these. Finally, Section 5 summarises our conclusions and gives some future research directions.

\section{Related Work}

Good surveys on VANET and intervehicle communication systems have been presented in the literature $[4,5,8]$. A good overview of recent research projects in this field is presented in [3], followed by a summary of the characteristics of standard wireless data links being integrated into vehicular systems, as well as a list of applications, their characteristics, and QoS requirements. A classification of vehicular applications, their requirements, characteristics, and related technical challenges are also presented in [9]. Several recent papers have discussed the challenges for the vehicle-to-vehicle wireless channel [10-12], which is very much affected by the high speed of cars, the vehicular traffic density, and obstacles like buildings.

A good tutorial on the IEEE standardisation activities in the field, mainly IEEE 802.11p and IEEE 1609.x, can be found in [13]. The system architecture, called Wireless Access in Vehicular Environments (WAVE), will support both traditional wireless communication using IPv6 as well as high-priority communication that uses a proprietary protocol called WAVE Short-Message Protocol (WSMP). IPv6 and WSMP communication is performed on two separate channels, where each unit supports multichannel operation. The European standardisation activities in the field, on the other hand, are described in [14]. The European ITS Communication Architecture supports three main vehicular application scenarios: traffic safety, traffic efficiency, and value-added services. The architecture supports both ad hoc communication with IEEE 802.11p and cellular communication with, for example, WiMAX or UMTS. There are two generic types of messages defined in the architecture. Cooperative Awareness Messages (CAM) provide the heartbeat, also called beaconing, information. Decentralised Environment Notification Messages (DENM) provide information about existing hazards in the defined area.

2.1. Selective Broadcast for Traffic Safety. Broadcasting techniques used in mobile and vehicular ad hoc networks are generally categorised as; (1) simple flooding, (2) probabilistic forwarding, (3) area based methods, and (4) methods using neighbour information [15]. In this section, we provide the reader with an overview of some of these protocols implemented in active traffic safety and emergency warning 
applications. Among the papers we summarise here, the ideas presented in $[2,16]$ form the basis of our evaluation, as presented later in Section 3.1.

The authors of [17] claim the main driver for network performance to be connectivity, and state that collisions at the medium access control (MAC) layer have a negligible effect on service reliability. So, they lay special emphasis on keeping the network connected. They create a highway scenario, where the warning service triggered in an emergency employs multiple broadcast cycles to guarantee the desired lifetime for the safety area. The next node to take over the message delivery task is chosen probabilistically. The authors develop an analytical model to predict the reliability of a single broadcast cycle. Following this model, it is possible to calculate the average number of nodes in a node's neighbourhood and derive the distribution of the space to be covered by the next message, so the forwarding probability can be fine-tuned accordingly. The simulation results presented validate the authors' analysis. However, an error-free wireless channel and constant vehicle density are assumed in the simulations; and sparse networks are not considered in the scenario.

Enhanced multihop vehicular broadcast (MHVB) is a flooding algorithm with special characteristics based on the position and speed of the cars [18]. It employs a detection mechanism using sensors for car traffic congestion, and lets the cars at the edges of a congestion send messages more frequently than those in the middle do. It also contains a method to suppress unnecessary packets, which is called the backfire algorithm. The algorithm defines a backfire region to pick the right node to retransmit a warning, namely the node farthest away from the sender. The other nodes in the backfire region cancel their scheduled transmission upon hearing the retransmission. In original MHVB, this is a circular region. In the enhanced version, the region becomes sectoral within a circle, enabling a directional backfire. Finally, a dynamic scheduling algorithm ensures that nodes farther away from the sender are allowed to transmit earlier than those closer to it. Simulations show, in terms of the success rate, that the enhanced version achieves better results than the original version.

Smart broadcast is a position-based protocol aiming at the maximisation of the one-hop progress of the alert message and the minimisation of the re-broadcast delay [19]. It is accompanied by a mathematical model providing a means to set the protocol's parameters optimally. It employs a contention resolution method to determine the next relay node at each hop. According to this, the source sends a request-to-broadcast. The nodes behind the source (in the desired direction for message propagation) enter a relay election phase. The coverage area is divided into sectors; and each sector is assigned a different contention window, the farther away from the source, the smaller. Each node picks a random backoff value from it's respective sector's window. So, a node far away from the source gets a short backoff value and becomes the first one to reply to the source with a clearto-broadcast. Upon hearing this message, the other nodes exit the contention phase. The source receives the reply, and sends the data. A comparison with 3 other broadcast protocols shows that smart broadcast has a competitive performance in terms of message propagation speed and one-hop progress.

Direction aware broadcast forwarding, a simple yet efficient method, is introduced [2] as an example of the communication protocols for cooperative collision avoidance in vehicle platoons. In their paper, the authors describe two versions of direction-aware broadcast. In naive broadcast, the vehicle detecting an emergency event starts sending warnings periodically. Upon receiving it, the other vehicles start sending their own periodical warnings if the message they received comes from their front. In intelligent broadcast with implicit acknowledgement, both the initiator as well as the repeaters cancel their periodical transmission when they hear the same warning coming from a node at their back. All receivers wait for a random time before starting to send their own warnings to see whether another node starts before them. If they do, they come to the conclusion that the warning has already propagated successfully, and do not start sending messages. The safety performance of these algorithms is evaluated through simulations; and the results show a significant improvement in terms of the success ratio under high background traffic and packet error rates when the intelligent version is used.

A simple selective broadcast algorithm, called edgeaware epidemic protocol, is introduced and evaluated [16]. According to this algorithm, only nodes at the boundary of a cluster of vehicles propagate messages, either instantly or in a store-and-forward fashion, thus keeping it alive when there is a disconnection in the network. Upon receiving a warning, nodes enter a random waiting period, the duration of which is inversely proportional with their distance to the sender of the message. During this period, they count the replicas they receive, in and against the desired flow of information separately, so they can use this information later to decide whether or not to forward the warning. This logic encourages those nodes closer to the edge of the sender's transmission range to relay the message, trying to increase the one-hop progress it makes. The author presents simulation results for three VANET scenarios with varying levels of connectivity. The channel model used in the simulations takes into account the effect of relative speed between two vehicles. The results confirm that the proposed protocol outperforms flooding in terms of successful information dissemination.

\subsection{Comparative Studies on Broadcast Techniques. The per-} formance of communication in VANET has been studied by a number of papers in the literature. In this section, we summarise some of these studies, covering various techniques like pure flooding, position-based flooding, positionbased unicast, one-hop broadcast, and more general data dissemination.

Three techniques used for multihop message propagation in VANET are evaluated in [20]: simple flooding, direction-aware flooding, and multipoint relaying (MPR, which originates from optimised link state routing (OLSR)). The authors are mainly interested in emergency situations in the immediate aftermath of a car crash and the extension 
of the safety area by information exchange between the cars. They design a scenario with a line of cars on a $1 \mathrm{~km}, 3$-lane motorway and conduct 3 sets of simulations with varying background data rates, number of cars, and probability errors to see the effect of background data traffic, vehicle density, and packet errors, respectively, on the performance. Probability errors are used to represent wireless channel conditions. The performance criteria of the study are delay, interpacket delay, warning delivery ratio, and overhead. The results favour flooding despite its large overhead.

In their study of 3 data dissemination techniques, the authors of [21] use a working prototype of a system for data gathering, dissemination, and visualisation. The dissemination techniques under investigation are same direction, opposite direction, and both directions. In each of these tecniques, data are broadcast periodically and nonselectively apart from the direction of the cars involved. The evaluation is based on a mathematical analysis of the broadcast utilisation, which is defined as the percentage of the newly covered area-that is, not covered previously-by a broadcast message, and on simulation. In addition to broadcast utilisation, latency time, accuracy in position estimation, and percentage of known vehicles are also used as performance criteria. Simulation results show that using vehicles moving in the opposite direction significantly improves the system's overall performance.

An investigation of various radio propagation models' impact on different VANET routing protocols is presented in [22]. Two deterministic radio propagation models, tworay ground reflection and line-of-sight differentiation, are implemented for this purpose. A third, detailed model, taking path loss, correlated shadowing, and multipath fading into consideration and simulating signal variability, is also implemented. These models are used to compare two unicast (greedy perimeter stateless routing, GPSR, and spatially aware routing $(\mathrm{SAR})$ ) and one broadcast (contention-based forwarding $(\mathrm{CBF})$ ) routing protocols, all multihop and position-based, through computer simulation in a Manhattan grid urban scenario with obstacles (buildings) under high car density. The main performance metric of the study is packet delivery, and it is shown that there are big differences between the different radio propagation models. Signal variability, in particular, reduces link reliability and, thus, needs to be taken into account in the simulations. The general conclusion to be drawn from this part of the study is that the broadcast protocol performs better than the unicast protocols. Analysing how the three protocols behave under different radio propagation models, the authors also come to the conclusion that protocol operation also changes significantly depending on the model used. Understanding these differences helps the prediction of the geographic packet distribution and, as a result, congestion control.

\section{Evaluation Scenario}

As we mentioned at the beginning of this paper, the purpose of this study is to evaluate a subset of selective broadcast algorithms in light of a realistic simulation environment, so we can observe and compare their performance with regard to mission-critical safety applications. The settings of such a simulation involve the selection of an appropriate mobility model, physical (PHY) and MAC layer, and scenario-related parameters like the number of nodes in the application's safety zone, the network density (as a representation of the application's penetration rate), node speed and the distance between nodes, the way the nodes enter and leave the network, the road length, the number of lanes, the direction of the vehicular traffic, the size and interval of the emergency messages, and the properties of the background data traffic. In the following sections, we first describe the broadcast algorithms as they are implemented in our simulations. Then, we summarise how we set our realistic scenario by carefully adjusting most of the parameters mentioned above.

3.1. Selective Broadcast under Inspection. In order to evaluate the efficiency of selective broadcast in vehicular safety applications, we implemented 4 algorithms, all with different heuristics. We call the first one naive broadcast (NB), and it is based on [2]. In this algorithm, upon encountering an emergency situation, the first vehicle initiates a periodic broadcast sequence and starts sending warning messages. Upon receiving a warning message, other vehicles start their own periodic broadcast sequence provided that the warning comes from a vehicle in front of them. Hence, they are called repeaters. There is no additional waiting time prior to the sending of the warnings and no termination condition for the periodic broadcast.

The second selective broadcast algorithm, also based on [2], is called intelligent broadcast (IB). In this more intelligent version, the first vehicle initiates its sequence as in naive broadcast, but it stops sending messages as soon as it overhears another vehicle at its back sending the same message, which is a sign showing that the warning has successfully propagated further down the road. The repeaters also start their sequence as in naive broadcast, but they, too, stop if they overhear others at their back repeating the warning. In addition to their periodic broadcast interval, the repeaters must also wait for a random duration $t_{\text {wait }}$, where $0 \leq t_{\text {wait }} \leq t_{\max }$ and $t_{\max }$ is the maximum waiting time, before sending their messages. If, while they are waiting, the stopping condition is satisfied, they cancel their sequence immediately since there is no need to repeat the warning any more.

For the third algorithm, we made a change, inspired by [23], to intelligent broadcast. So, we call it modified intelligent broadcast (MIB). In this version, we put some more intelligence to the waiting time $t_{\text {wait }}$ introduced by the repeaters prior to sending their warnings. Instead of determining $t_{\text {wait }}$ randomly, we made it inversely proportional to the distance between the repeater and the vehicle it has just received the message from. The new waiting time is formulated as

$$
t_{\text {wait }}=t_{\max }\left(1-\frac{d}{r_{\text {trans }}}\right) .
$$

In (1), $r_{\text {trans }}$ is the maximum transmission range. Defining the waiting time like this gives priority to the vehicles 
TABLE 1: Simulation parameters.

\begin{tabular}{ll}
\hline Description & Value \\
\hline $\begin{array}{l}\text { Number of vehicles (variable) } \\
\text { Wireless transmission range } \\
(\text { variable) }\end{array}$ & $20 \cdots 100$ \\
\hline Emergency warning size & $100 \mathrm{~m} \cdots 500 \mathrm{~m}$ \\
Emergency warning interval & $100 \mathrm{~B}$ \\
Background message size & $100 \mathrm{~ms}$ \\
Background message interval & $800 \mathrm{~B}$ \\
Wait-before-send time & $500 \mathrm{~ms}$ \\
$($ minimum $\cdots$ maximum) & $0 \mathrm{~ms} \cdots 10 \mathrm{~ms}$ \\
\hline $\begin{array}{l}\text { Highway segment length } \\
\text { Highway width in one direction }\end{array}$ & $2000 \mathrm{~m}$ \\
Highway lane width & $14 \mathrm{~m}$ \\
Vehicle speed & $3.5 \mathrm{~m}$ \\
$($ minimum $\cdots$ maximum) & $60 \mathrm{~km} / \mathrm{h} \cdots 120 \mathrm{~km} / \mathrm{h}$ \\
Deceleration rate & $4 \mathrm{~m} / \mathrm{s}^{2}$ \\
Reaction time & $1.6 \mathrm{~s}$ \\
\hline
\end{tabular}

farther away from the sender over the ones closer to it, resulting in a probabilistically fuller exploitation of the wireless transmission range and, thus, in the full coverage of the emergency zone in fewer steps.

Our fourth algorithm, originally proposed in [16], can actually be considered another variant of the three above. It is called epidemic broadcast (EB). According to this algorithm, the initiator and the repeaters work as in modified intelligent broadcast. The waiting time $t_{\text {wait }}$ is selected randomly between 0 and $t_{\max }$, where $t_{\max }$ is chosen by a formula exponentially biased towards vehicles farther away from the sender. We simplified that formula and redefined the waiting time as follows:

$$
t_{\text {wait }}=t_{\max } \cdot \exp \left(-\frac{d}{r_{\text {trans }}}\right) .
$$

During each broadcast interval, extended by the waiting time, the repeaters count the duplicate messages they receive from their front and their back. At the end of the waiting time, they enter a decision process instead of immediately sending their warning message. So, the forwarding decision at the end of each interval is based on the difference between the counted messages that far. The vehicles with a bigger difference have a higher probability of keeping the message alive, denoted by $P_{\text {send }}$ and formulated in our implementation as follows:

$$
P_{\text {send }}=\exp \left(-\frac{w_{\text {back }}}{w_{\text {back }}+w_{\text {front }}}\right) .
$$

In (3), $w_{\text {back }}$ and $w_{\text {front }}$ are the number of duplicate messages received from the back and front, respectively. Thus, the decision process favours those nodes with an unbalanced message count, which means they are closer to the edge of the sender's transmission range.

As a benchmark for the algorithms above, we included a simple flooding algorithm (FL) to our evaluation. According to this algorithm, the initiator starts a periodic sequence, and the repeaters start their own as soon as they receive their first warning. Like in the naive broadcast algorithm, there is no termination condition for the sending of the periodic warnings.

Almost all broadcasting methods proposed so far use some sort of position information [7]. In our simulation study, we therefore also assume every node to be aware of its own position and able communicate that information to the others. Various other information, like a vehicle's own type, speed, direction, and destination, can be generally considered available as well. Although not used in our evaluation, this type of secondary information is quite useful for a number of vehicular applications. Finally, background traffic is generated by a simple application issuing periodic, single-hop broadcast messages with the parameters given in Table 1 . The background application runs independently on each node of the vehicular network, and a node stops its background application as soon as it receives its first emergency warning.

Figure 1 demonstrates in a simplified manner how selective broadcast generally works. In order to keep the example uncomplicated, let us assume that the vehicles implement intelligent broadcast for the emergency warning application. The course of events differ, of course, when one of the other algorithms described above is employed; but it is easy to follow their logic using this example as well. (a) Vehicle 1 encounters an emergency, initiates its periodic warning sequence, and broadcasts a message. The packet is received by the other vehicles within transmission range, that is, vehicles 2,3 , and 4 in our example. Upon reception, vehicles 2, 3, and 4 all enter a waiting phase of random duration. The vehicle with the shortest waiting time starts its own periodic broadcast sequence once the waiting phase is over. In our example, this is vehicle 4 . (b) As vehicle 4 broadcasts its message, vehicles 2 and 3 overhear the transmission and, realising that this is the same emergency warning they are about to send, they cancel their own transmission since it is not necessary anymore. Vehicle 1, the initiator of the emergency warning, also stops its periodic broadcast sequence upon overhearing the transmission, because this is an implicit acknowledgement showing that the warning has successfully propagated backwards, that is, in the desired direction. In the meantime, vehicles $5,6,7$, and 8 receive the warning for the first time. (c) The same procedure applies for the next step, that is, vehicles $5,6,7$, and 8 wait for a random duration before propagating the warning. In our example, it is vehicle 7 now which has the shortest random waiting time, so it starts its periodic broadcast sequence as the waiting time is up. Receiving the warning, vehicle 4 cancels its sequence since it now knows that the warning has propagated backwards, whereas vehicles 5,6 , and 8 decide not to start their respective sequences since another vehicle within their neighbourhood already did it for them.

3.2. Simulation Settings. As our tool for discrete event simulation, we have chosen ns-3 [24], which is developed as a free software licensed under GNU GPLv2 to replace 


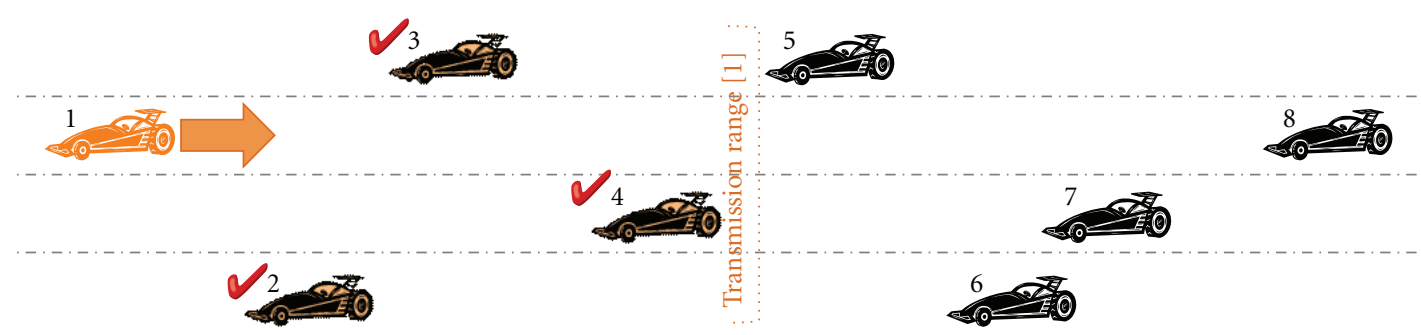

(a)

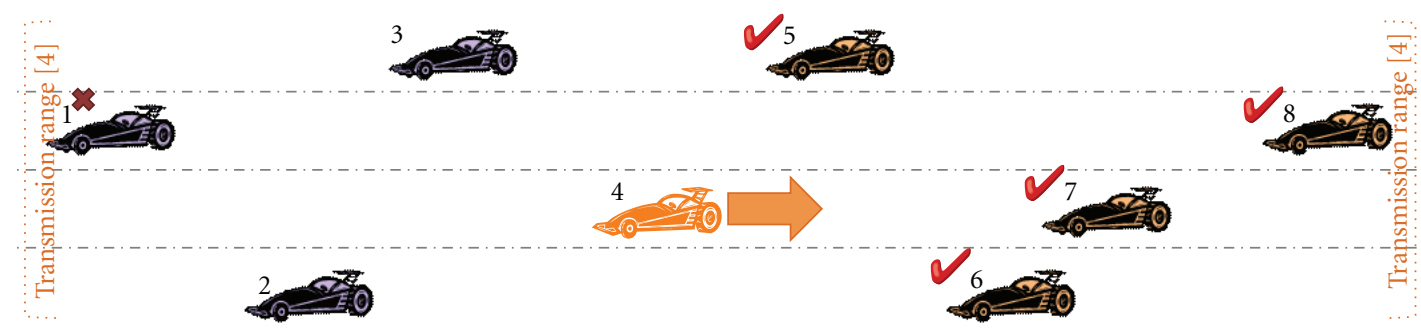

(b)

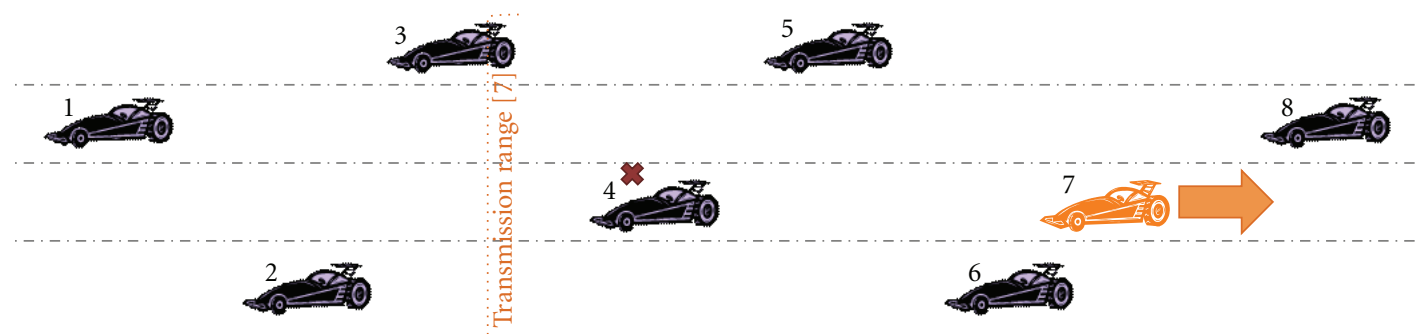

(c)

FIGURE 1: A demonstrative example showing three snapshots from a highway scenario with a 4-lane road segment and 8 cars: (a) periodical emergency warning broadcast is initiated by car 1 . The warning is received by cars 2, 3, and 4. (b) Car 4 propagates the message. Car 1 overhears this and cancels its own broadcast sequence. Cars 5, 6, 7, and 8 receive the warning. (c) Car 7 propagates the message. Car 4 overhears it and cancels its own sequence.

eventually the very popular ns-2 [25]. ns-3 has already most of the models and functions of its predecessor and, like ns2 , is highly trusted among the network research community. Table 1 summarises the parameters and their values as we used them in our simulations.

The simulation parameters and additional settings we chose for our simulations are the result of a synthesis of various previous studies. For instance, the authors of [8] utilise periodic, one-hop broadcast for safety warnings with 10 messages per second, a maximum latency of $100 \mathrm{~ms}$, and $150 \mathrm{~m}$ transmission range. They use IEEE 802.11p, orthogonal frequency-division multiplexing (OFDM), a $10 \mathrm{MHz}$ channel, and a data rate of 3-27 Mbps. In [2], the authors define 50 vehicles moving at $32 \mathrm{~m} / \mathrm{s}$, packets of $64 \mathrm{~B}$, a warning period of $100 \mathrm{~ms}$, and $80-800 \mathrm{Kbps}$ background traffic. They use 802.11 a, prioritise the emergency communication, and introduce channel errors. Finally, the authors of [20] run simulations with 24, 45, and 60 nodes, all moving at $130 \mathrm{~km} / \mathrm{h}$, with an intervehicle distance of 130,70 , and $50 \mathrm{~m}$, respectively. The emergency traffic is periodic with 10 messages per second, and the packet size is $100 \mathrm{~B}$. They also generate single-hop, constant bit rate (CBR) background traffic at rates of 5, 10, and 20 packets per second with $1 \mathrm{~KB}$ packets. Their error probabilities are 0, 10, and 25\%. Other parameters they use include a transmission range of $250 \mathrm{~m}$, a carrier sense range of $550 \mathrm{~m}$, a data rate of $11 \mathrm{Mbps}$, and 40 seconds of simulated time. Our target scenario represents a highway with $n$ mobile nodes, that is, vehicles, configured as explained below.

3.2.1. Node Positioning. All vehicles are assigned a random $x$ coordinate on the highway, chosen uniformly from the full length of the segment, as their initial position. They are also assigned a $y$ coordinate; these, however, are chosen from integer multiples of the lane width and may not exceed the road width. The $(x, y)$ coordinates thus put each vehicle precisely on a single lane along the highway segment. There are only two exceptions to this positioning strategy. The first one is the emergency initiator, that is, the first node to encounter the emergency situation and initiate the broadcast sequence. This node is placed on the first lane at the beginning of the highway segment. The second exception is one node put on the first lane at the very end of the highway segment, ensuring that the last node to receive a warning always has the same distance from the emergency initiator 
and, thus, yielding a fairer comparison between the broadcast algorithms we evaluate. Obviously, it is very important to us to have a realistic node distribution on the road. With the highway segment and lane dimensions given in Table 1, the probabilistic average of the intervehicle distance is $80 \mathrm{~m}$ for 100 nodes. According to vehicular ad hoc routing studies like [26], this is a realistic value for sparse vehicular networks like the ones formed on a highway.

3.2.2. Mobility. Since, within the scope of this study, we are interested in the immediate aftermath of an emergency situation, spanning typically a few seconds, it is sufficient for our purposes to take a snapshot of the vehicular network as the warning message starts to propagate, rather than simulating the vehicular mobility in great detail. Furthermore, our work does not investigate the driver behaviour after having received the early warning message in an emergency situation, which means that the changes in mobility due to driver reactions do not need to be a part of the simulation. Thus, in our simulations, we employ a simple mobility model called constant velocity. In this model, a uniformly random individual speed between the minimum and maximum values given in Table 1 , which does not change during the entire simulation, is assigned to each vehicle. There are also no changes in direction. It is, of course, possible to implement a more sophisticated vehicular mobility model and fine-tune further the simulation settings, such as minimum intervehicle distances or lane changing. Although interesting, these elements are currently out of scope for our study.

3.2.3. Wireless Module. Most PHY proposals are based on IEEE 802.11, and random access protocols (like carrier sense multiple access (CSMA)) are preferred to controlled access protocols (like time division multiple access (TDMA)) [4]. Furthermore, it is generally accepted that dedicated shortrange communications (DSRC) may not be appropriate for all application types [15]. Therefore, we used the $802.11 \mathrm{p}$ standard with $5 \mathrm{GHz}$ frequency range and $10 \mathrm{MHz}$ data rate at $6 \mathrm{Mbps}$ at the physical layer. As far as channel modelling is concerned, the current focus is on IEEE 802.11 DCF with one-hop broadcast as the basic communication type, by which MAC becomes simple CSMA. Packet reception is influenced by vehicle density, radio channel conditions, data rate, transmit power, and contention window size [8]. Two approaches can be distinguished in developing channel models. First, there are statistical models, which are faithful emulations of variations in channel behaviour in time. Second, there are deterministic models, doing exact estimations of the small scale channel fading characteristics at particular points in space and time [11]. For our purposes, we need a channel model adopting the first approach. Our wireless channel operates on the constant speed propagation delay model and log-distance propagation loss model available in ns-3. Finally, the MAC layer we chose has 4 application QoS classes with respective queues, where best effort is the default.

\section{Simulation Results}

In this section, we compare the performance of the four algorithms summarised above and of flooding, against a unified, realistic highway scenario. We first describe the performance criteria we consider important for the evaluation of a traffic safety application like collision avoidance. The results we achieved are presented and interpreted after that.

4.1. Performance Criteria. There are a number of performance metrics in the literature, such as packet error, loss, or delivery ratios, end-to-end delay, normalised network load, and packet duplication [27], which are typically more common for an evaluation from a pure network performance point of view. Nevertheless, we want to put more emphasis on the network's performance with respect to the missioncritical nature of the safety application running on top of it. Thus, we introduce four new performance criteria for our evaluation. We believe these will provide the reader with a clearer understanding of the various algorithms' success in fulfilling the stringent reliability requirements of the safety application. The criteria we are interested in are the following.

4.1.1. Warning Effectiveness. In an emergency situation like the possible collision of vehicles, it is of extreme importance that the application in charge of delivering the warning does the right thing. Thus, the effectiveness metric is defined as the percentage of the vehicles having received the collision warning in a timely manner at the end of the simulation. We measure this phenomenon by keeping track of the distance between the point where the emergency is initialised and the location of the vehicle as it receives the warning for the first time. Based on the emergency distance information we derive for the new recipient of the warning, and also given its speed, deceleration and driver reaction time as in $[1,2]$, we can determine whether a vehicle has received the warning before it is too late. Within the scope of our study, a timely delivery (i.e., before it is too late) of the emergency warning means that, by the time it receives the warning, the recipient's distance to the accident location is sufficient to stop in time.

4.1.2. Warning Efficiency. This metric is about evaluating the algorithms' ability to do things right. It is measured by observing two phenomena. The first one is the number of messages generated per vehicle until all reachable vehicles have been warned. If all the vehicles in the network can actually be reached before the simulation ends, this number gives us a per-vehicle average of the number of required warnings for a particular algorithm to cover completely the safety area. Otherwise, it gives us the average number of warnings to be sent by each vehicle to reach all the reachable vehicles. Depending on the different algorithm's heuristics, however, more messages may or may not be issued even after the last reachable vehicle has been informed on the emergency situation. This is represented by the second phenomenon we observe, that is, the total number of messages generated per vehicle until the end of the 


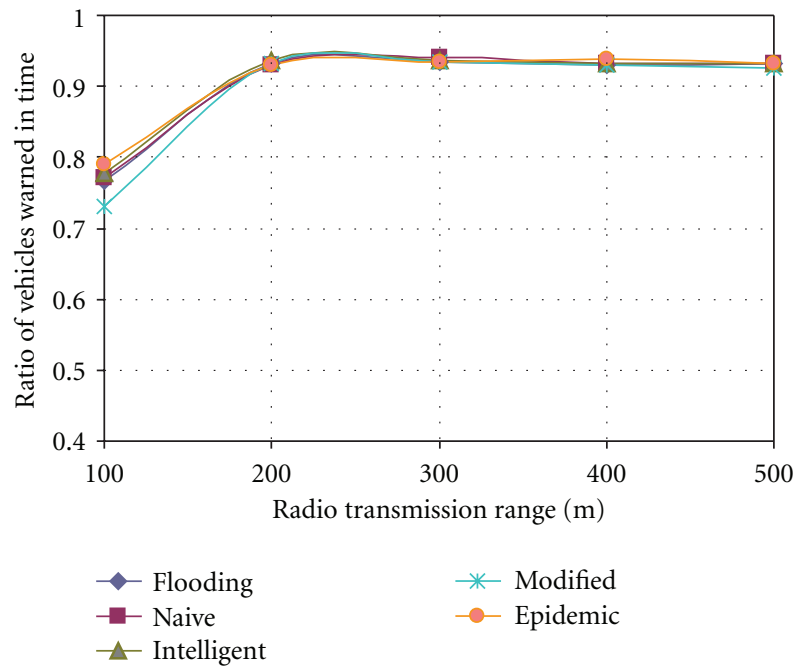

(a)

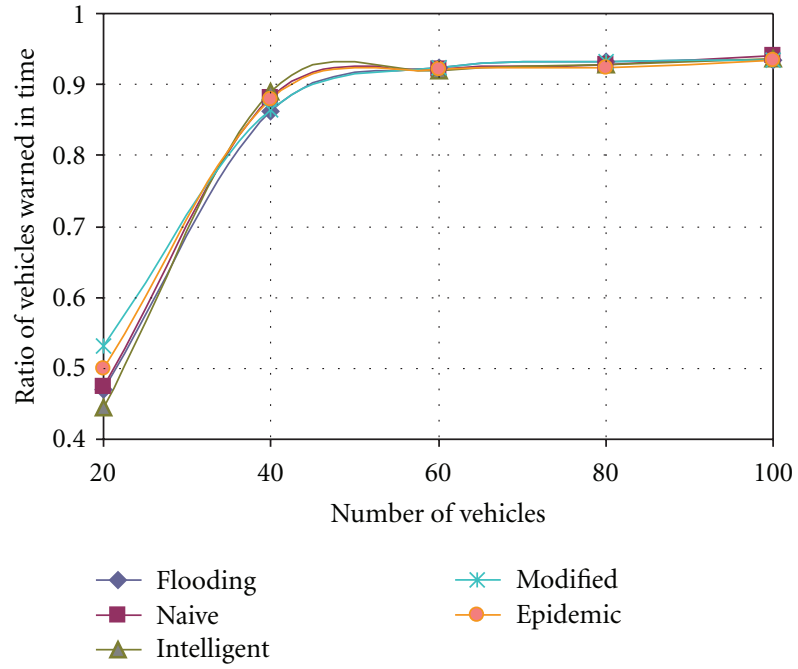

(b)

FIGURE 2: Warning effectiveness results, showing the algorithms' performance in terms of the ratio of vehicles warned in time as a function of (a) the transmission range for 100 vehicles and (b) the number of vehicles for $300 \mathrm{~m}$ transmission range.

simulation. Obviously, it is important to have a low value for the number of required messages; but it is also important to have a low value for the actual number of generated messages, which shows that the algorithm stops generating unnecessary data traffic soon enough after the last reachable vehicle has been warned.

4.1.3. Warning Propagation. The time required to reach all vehicles, or the last reachable vehicle in case not all are reachable, is the time for the algorithm to complete. So the propagation metric gives us an idea about how quickly the warning messages are disseminated throughout the vehicular network and, thus, how quickly the algorithm converges. It is easy to derive from this value the one-hop progress the packets make, which is another interesting value showing whether the algorithm can make full, or at least efficient, use of the nodes' wireless transmission range. The warning propagation time can be used as a criterion to see the effect of time of day and market penetration, both of which being represented by node density, on the success of the algorithms.

4.1.4. Warning Overhead. Based on the volume of the traffic generated by the broadcast algorithm, other network applications may be affected by the safety warning application in various levels. We observe this by generating background traffic, which represents those other applications, and measuring its packet delivery rate in the face of each of the broadcast algorithms it encounters. In our simulations, a packet is dropped at the wireless physical layer due to channel switching, collision, too small power, or random noise. The results give us an idea about the impact of our safety application on the rest of the network. The effect in the reverse direction, of course, is maybe even more important but, since our emergency message traffic is prioritised, this is beyond the scope of the study presented in this paper.
4.2. Comparison of Algorithms. In order to evaluate the algorithms we implemented, we conducted ns-3 simulations using the parameters set as shown in Table 1. We used the radio transmission range and the number of nodes in the network as our simulation variables. As we changed the transmission range between $100 \mathrm{~m}$ and $500 \mathrm{~m}$, we fixed the number of nodes at 100 . As we varied the number of nodes between 20 and 100, we set the transmission range to $300 \mathrm{~m}$. This way, we were able to see the performance of the broadcast algorithms we implemented from two different perspectives, both of which we find important. We ran the simulations 100 times, all for $5 \mathrm{~s}$ with different random number seeds, for each algorithm described in Section 3.1 and each different value of the chosen variables. Each data point in the graphics is, thus, the average of 100 individual values, minimising the possibility of a biased result. In each simulation, background traffic starts immediately, whereas the first emergency warning is initiated $1 \mathrm{~s}$ into the simulation.

4.2.1. Warning Effectiveness. Figure 2 shows the performance of the algorithms in terms of warning effectiveness, described in Section 4.1, as we change (a) the transmission range, and (b) the number of nodes. Here, our criterion is the ratio of vehicles which receive the emergency warning in time, that is, when they still have a distance sufficient to stop before they collide with the car at the emergency site, taking into account the driver's reaction time as well as the car's deceleration rate.

According to Figure 2(a), all algorithms show a similar performance, especially for transmission ranges higher than $200 \mathrm{~m}$. The ratio of cars they reach in time is above 0.90 . The ongoing background communication between the nodes not yet received the warning causes some of the emergency packets to be dropped, and the low transmission range prevents the network from having the redundancy which 
could enable the overall system to tolerate these losses. For transmission ranges as low as $100 \mathrm{~m}$, all the algorithms suffer from too low network connectivity. From Figure 2(b) we can see the same trend. In sparse networks, all algorithms have a relatively low performance, that is, between 0.45 and 0.55 ; but they manage a success ratio as high as 0.90 as the network density increases.

4.2.2. Warning Efficiency. Figure 3 presents the results for warning efficiency, described in Section 4.1, as we change our two variables. This time, our criterion is the number of messages generated by each node in each algorithm in order to achieve the effectiveness shown in Figure 2, that is, the price paid for the above performance. Here, we see a clear distinction between the algorithms with and without selection criteria, that is, the ones incorporating a selective logic into their broadcast mechanism and the ones flooding the network. Typical values for the former group are around 1, whereas it is 39 for the latter. Here, it should also be noted that the algorithms with higher numbers of generated messages per node, that is, flooding and naive broadcast, also lack a stopping condition for their periodic broadcast, whereas the other two with very low numbers of generated messages per node, that is, intelligent and modified intelligent broadcast, benefit from their intelligent decision mechanisms for both when to broadcast a message and when to stop broadcasting. Epidemic broadcast, with its decision criterion based on the difference between the number of messages received from and against the direction of the desired warning flow, shows a mediocre performance in terms of efficiency. The main reason behind this performance is that, due to the small emergency messaging interval, the difference mentioned above is also quite small, resulting in an approximately $50 \%$ chance of relaying the warnings and increasing the number of messages per vehicle.

The number of generated warnings gives us a good idea about the cost of the broadcast algorithm's performance; but that is not all. In many cases, the number of warning messages really required to reach all reachable vehicles is less than those actually generated. In other words, the number of messages needed to warn all nodes about the emergency is usually smaller than what is shown in Figure 3. For the algorithms without a stopping condition, that is, flooding and naive broadcast, the reason for this is obvious: they just keep sending their periodical warnings. For the others, that is, intelligent, modified intelligent, and epidemic broadcast, we can say that it takes some time for the stopping condition to take action. Moreover, this condition is based on the re-reception of a warning by its sender as the message is relayed by the vehicles behind it. Thus, the condition is never met for the vehicles at the end of the vehicle cluster. Typically, the last vehicle in the safety zone defined in the simulation never stops sending periodic messages since it never overhears its own message relayed by another vehicle behind it. Thus, it is also a good idea to have a look at the difference between the number of generated and actually required (i.e., useful) messages to achieve the same warning performance.

Figure 4 shows the number of messages per vehicle actually required to cover the safety area, as we change (a) the transmission range and (b) the number of nodes. Like the results presented in the previous figure, there are three distinct groups, one with relatively high values, the other with low values, and the third with values in between. The higher values belong to flooding and naive broadcast, and it is interesting to see that, for transmission ranges higher than $200 \mathrm{~m}$, the averages are around 2 messages per node, which are much lower than the averages in the previous figure. The lower values, on the other hand, belong to intelligent and modified intelligent broadcast, that is, those algorithms with stopping conditions, and the averages are very close to those in the previous figure. Epidemic broadcast, again, is placed between these two groups. The results show that a well-defined stopping condition can decrease significantly the actual cost of the emergency broadcast application by minimising the number of unnecessary warning repetition.

It is also interesting to see that, in Figure 4(b) and for flooding and naive broadcast, the number of useful warnings increases as the number of vehicles is increased from 20 to 60 and decreases again from 60 nodes to 100 . The reason for that is that the network is largely disconnected when the network density is low; and the corresponding warning success rate is around 0.5, as can be seen from Figure 2(b). Due to the low number of nodes being successfully warned, the number of useful warnings also remains low. As the network becomes denser, the number of required warnings increases with the success rate. As the network becomes even denser, the success rate stabilises but it becomes possible to maintain the same rate with fewer messages due to the significant increase in the number of neighbours that can benefit from a single warning.

4.2.3. Warning Propagation. Figure 5 shows the time each algorithm requires to deliver the emergency warning to the last reachable vehicle in the safety area. As two of the algorithms we implemented, namely, modified intelligent and epidemic broadcast, try to maximise the one-hop propagation of the warning messages by setting waiting times inversely proportional to the distance between the relay node and its sender, it is particularly insightful to observe the results they achieved. According to the figure, flooding and naive broadcast do not perform as well as the other three algorithms. In other words, their average propagation time for transmission ranges higher than $200 \mathrm{~m}$ is around $1.2 \mathrm{~s}$ to $1.3 \mathrm{~s}$, whereas epidemic broadcast ranks between $1.1 \mathrm{~s}$ and $1.2 \mathrm{~s}$, and intelligent as well as modified intelligent broadcast have an average value well below 1.1 s. An interesting result in Figure 5 is that there is no significant difference in propagation time between intelligent and modified intelligent broadcasting, keeping in mind that the only difference between these algorithms is that the former defines a random waiting time prior to relaying a warning, whereas the latter employs a waiting time inversely proportional to distance. 


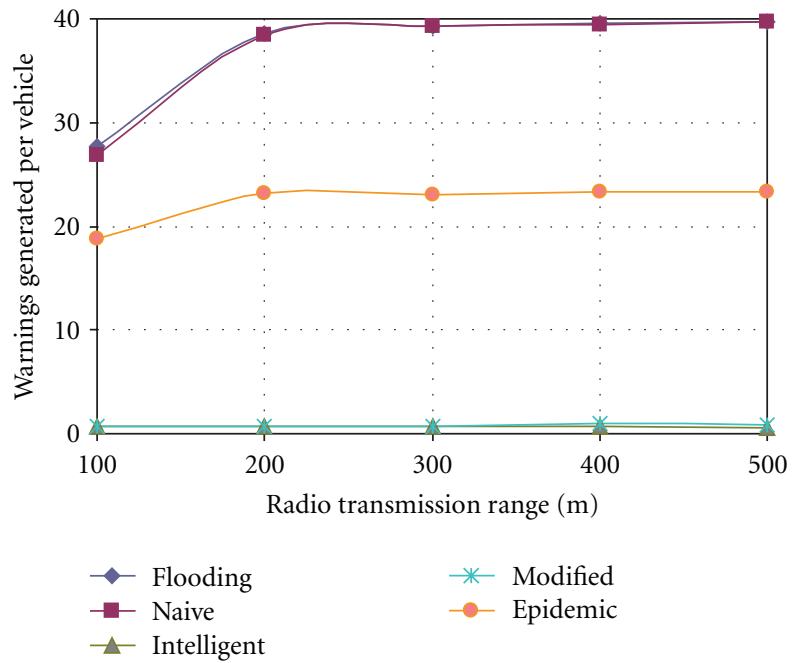

(a)



(b)

FIGURE 3: Warning efficiency results, showing the algorithms' performance in terms of the number of warnings generated per second by each vehicle as a function of (a) the transmission range for 100 vehicles and (b) the number of vehicles for $300 \mathrm{~m}$ transmission range.

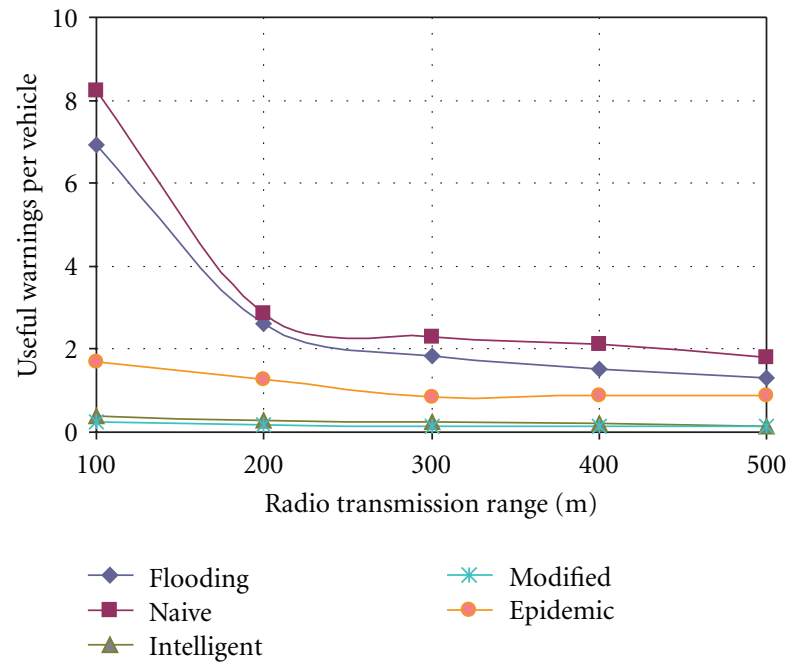

(a)



(b)

FIGURE 4: Warning efficiency results continued, showing the algorithms' performance in terms of the number of useful warnings (i.e., actually required to warn a new node) generated per second by each vehicle as a function of (a) the transmission range for 100 vehicles and (b) the number of vehicles for $300 \mathrm{~m}$ transmission range.

4.2.4. Warning Overhead. Finally, we observe the effect of the data traffic generated by the emergency warning application on other applications likely to be running in the network at the same time. Even though the emergency warning application has higher priority, that is, nodes are supposed to cease all other traffic as soon as they receive their first warning message, at any given time, there will be nodes in the network not having received the warning yet. Thus, these nodes' background data traffic is bound to be affected by the overhead created by the emergency application, and we need to evaluate this. Figure 6 shows the delivery ratio of the packets belonging to the background applications. The results show that all the emergency broadcast algorithms we tested have a similar impact on the background traffic, which seems counterintuitive. Given that they all incur different amounts of overhead on the network, as shown in Figure 3, one would expect to see a significant difference here. However, the frequency of the background traffic generation is much lower than that of the emergency application, and the propagation of the emergency warning is relatively fast, so that most of the background traffic is indeed ceased very quickly and regardless of the overhead of the emergency application. On the other hand, the background data's successful delivery rate decreases drastically as the 




(a)

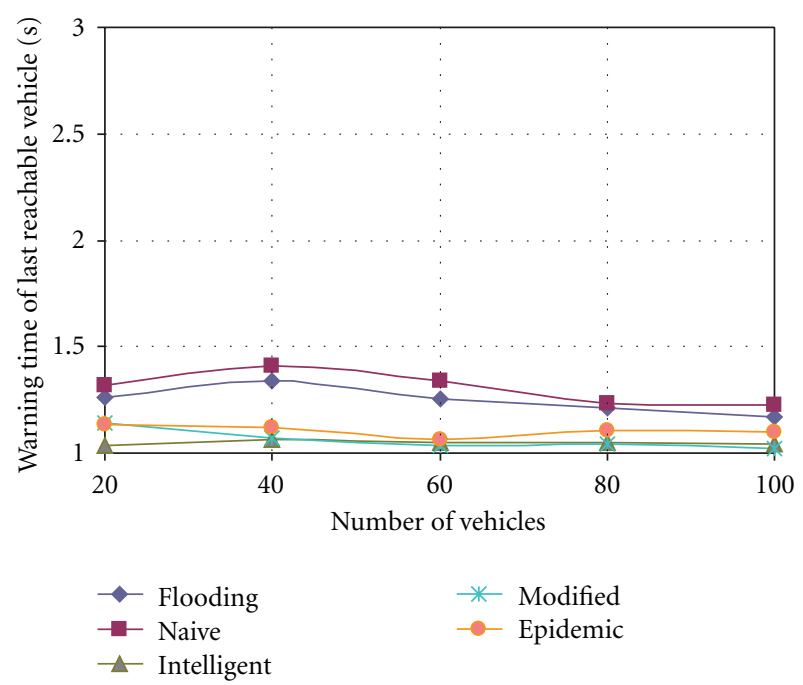

(b)

FIGURE 5: Warning propagation results, showing the algorithms' performance in terms of the time required to inform the last reachable vehicle as a function of (a) the transmission range for 100 vehicles, and (b) the number of vehicles for $300 \mathrm{~m}$ transmission range.



(a)



(b)

FIGURE 6: Warning overhead results, showing the algorithms' performance in terms of the ratio of background application data packets successfully delivered per vehicle as a function of (a) the transmission range for 100 vehicles and (b) the number of vehicles for $300 \mathrm{~m}$ transmission range.

transmission range increases from $100 \mathrm{~m}$ to $300 \mathrm{~m}$ or the number of nodes increases from 20 to 100 , due to more nodes sharing the wireless spectrum.

\section{Conclusion}

Emergency warning applications using VANET have gained great interest as a powerful means of improving road safety. However, their time- and life-critical field of operation requires high QoS standards in terms of reliable, timely, and guaranteed message delivery. As mentioned in the introduction, the key to the successful dissemination of safety warnings is the mechanism to decide when to start and stop message relaying. The algorithms developed for information dissemination in VANET must meet these requirements so we can use them for these applications. In this paper, we compare the performance of a subset of selective broadcast algorithms in a unified simulation scenario, that is, under the same conditions, to see how well they cope with the criteria mentioned above. 
For our evaluation, we use four new performance metrics to measure the effectiveness, efficiency, timeliness, and overhead of the algorithms. The results we obtain favour selective broadcast algorithms with intelligent decision mechanisms regarding their starting and stopping conditions. The results can be further interpreted as follows. First, periodicity improves significantly the success rate of the algorithm, due to the fact that, by creating redundancy, it helps the vehicular network to tolerate eventual packet losses. In an emergency scenario, especially when the transmission range is not high, it is simply too risky for a node to broadcast a warning only once. Second, it is more important to have some selection criteria (at all) about when to start relaying emergency messages than to employ the most sophisticated mechanism optimising the selection in question. A simple, random wait-before-send logic can achieve the same performance as a more complex, distance-based logic formulated by exponential functions, as confirmed by the fact that there is no significant difference in the performance of intelligent and modified intelligent broadcast. Finally, the stopping condition is not less important than the starting condition. If they employed stopping conditions, algorithms like flooding and naive broadcast would have better efficiency and overhead results.

The results we present show that much work remains to be done to realise safety applications with 100\% reliability, so they can be widely and safely used in vehicles. Our evaluation is a contribution to the efforts in this direction; the results we obtain from our simulations help us to understand better the design requirements of a high-performance selective broadcast algorithm. It is necessary, on the other hand, to improve the simulation itself in order to achieve a platform which can evaluate the algorithms under investigation as fairly as possible, producing results as close to the real world as possible. For instance, the effect of background traffic on the emergency messages can be investigated for situations in which there are no application priorities. More accurate mobility models can be incorporated into the simulations, containing elements for changing lanes, keeping the safety distance, platooning, and driver behaviour. Concerning the wireless communication infrastructure, more realistic PHY conditions can be made part of the simulation if appropriate channel error and propagation loss models are included. Our research plan for the near future is to address some of these issues and, currently, we are working on an adaptive selective broadcast algorithm and the integration of a new propagation loss model based on real-life measurements into our simulations.

\section{Acknowledgment}

This work is partly funded by Excellence Center at Linköping-Lund in Information Technology (eLLIIT). K. Bür and M. Kihl are members of Lund Center for Control of Complex Engineering Systems (LCCC), a Linnaeus Center at Lund University, funded by the Swedish Research Council. M. Kihl is partly funded by the VINNMER program at the Swedish Governmental Agency for Innovation Systems (VINNOVA).

\section{References}

[1] P. L. Olson and M. Sivak, "Perception-response time to unexpected roadway hazards," Human Factors, vol. 28, no. 1, pp. 91-96, 1986.

[2] S. Biswas, R. Tatchikou, and F. Dion, "Vehicle-to-vehicle wireless communication protocols for enhancing highway traffic safety," IEEE Communications Magazine, vol. 44, no. 1, pp. 74-82, 2006.

[3] P. Papadimitratos, A. La Fortelle, K. Evenssen, R. Brignolo, and S. Cosenza, "Vehicular communication systems: enabling technologies, applications, and future outlook on intelligent transportation," IEEE Communications Magazine, vol. 47, no. 11 , pp. 84-95, 2009.

[4] Y. Toor, P. Muhlethaler, and A. Laouiti, "Vehicle ad hoc networks: applications and related technical issues," IEEE Communications Surveys and Tutorials, vol. 10, no. 3, pp. 74$88,2008$.

[5] M. Kihl and M. Sichitiu, "Inter-vehicle communication systems: a survey," IEEE Communications Surveys and Tutorials, vol. 10, no. 2, pp. 88-105, 2008.

[6] F. Li and Y. Wang, "Routing in vehicular ad hoc networks: a survey," IEEE Vehicular Technology Magazine, vol. 2, no. 2, pp. 12-22, 2007.

[7] W. Chen, R. K. Guha, T. J. Kwon, J. Lee, and I. Y. Hsu, "A survey and challenges in routing and data dissemination in vehicular ad-hoc networks," in Proceedings of IEEE International Conference on Vehicular Electronics and Safety, pp. 328333, Columbus, Ohio, USA, 2008.

[8] H. Hartenstein and K. P. Laberteaux, "A tutorial survey on vehicular ad hoc networks," IEEE Communications Magazine, vol. 46, no. 6, pp. 164-171, 2008.

[9] M. Kihl, "Vehicular network applications and services," in Vehicular Networks: Techniques, Standards, and Applications, CRC Press, 2009.

[10] A. F. Molisch, F. Tufvesson, J. Karedal, and C. F. Mecklenbräuker, "A survey on vehicle-to-vehicle propagation channels," IEEE Wireless Communications, vol. 16, no. 6, pp. 12-22, 2009.

[11] D. W. Matolak, "Channel modeling for vehicle-to-vehicle communications," IEEE Communications Magazine, vol. 46, no. 5, pp. 76-83, 2008.

[12] C. X. Wang, X. Cheng, and D. Laurenson, "Vehicle-to-vehicle channel modeling and measurements: recent advances and future challenges," IEEE Communications Magazine, vol. 47, no. 11, pp. 96-103, 2009.

[13] R. Uzcategui and G. Acosta-Marum, "Wave: a tutorial," IEEE Communications Magazine, vol. 47, no. 5, pp. 106-133, 2009.

[14] T. Kosch, I. Kulp, M. Bechler, M. Strassberger, B. Weyl, and R. Lasowski, "Communication architecture for cooperative systems in Europe," IEEE Communications Magazine, vol. 47, no. 5, pp. 116-125, 2009.

[15] X. X. Diao, J. J. Li, K. M. Hou, H. Y. Zhou, and A. Jacquot, "Cooperative inter-vehicle communication protocol dedicated to intelligent transport systems," in Proceedings of the New Technologies, Mobility and Security Conference and Workshops (NTMS '08), pp. 1-5, Tangier, Morocco, November 2008.

[16] M. Nekovee, "Epidemic algorithms for reliable and efficient information dissemination in vehicular ad hoc networks," IET Intelligent Transport Systems, vol. 3, no. 2, pp. 104-110, 2009. 
[17] R. Fracchia and M. Meo, "Analysis and design of warning delivery service in intervehicular networks," IEEE Transactions on Mobile Computing, vol. 7, no. 7, pp. 832-845, 2008.

[18] M. Mariyasagayam, T. Osafune, and M. Lenardi, "Enhanced Multi-Hop Vehicular Broadcast (MHVB) for active safety applications," in Proceedings of International Conference on Intelligent Transport Systems Telecommunications, pp. 1-6, Sophia Antipolis, France, 2007.

[19] E. Fasolo, A. Zanella, and M. Zorzi, "An effective broadcast scheme for alert message propagation in vehicular ad hoc networks," in Proceedings of IEEE International Conference on Communications, vol. 1, pp. 3960-3965, Istanbul, Turkey, 2006.

[20] P. Muhlethaler, A. Laouiti, and Y. Toor, "Comparison of flooding techniques for safety applications in VANETs," in Proceedings of International Conference on Intelligent Transport Systems Telecommunications, pp. 1-6, Sophia Antipolis, France, 2007.

[21] T. Nadeem, P. Shankar, and L. Iftode, "A comparative study of data dissemination models for VANETs," in Proceedings of International Conference on Mobile and Ubiquitous Systems, pp. 1-10, San Jose, Calif, USA, 2006.

[22] R. Bauza, J. Gozalvez, and M. Sepulcre, "Operation and performance of vehicular ad-hoc routing protocols in realistic environments," in Proceedings of IEEE Vehicular Technology Conference, pp. 1-5, Calgary, Canada, September 2008.

[23] L. Briesemeister and G. Hommel, "Role-based multicast in highly mobile but sparsely connected ad hoc networks," in Proceedings of Mobile and Ad Hoc Networking and Computing, pp. 45-50, Boston, Mass, USA, 2000.

[24] The ns-3 network simulator, 2010, http://www.nsnam.org/.

[25] The network simulator ns-2, 2010, http://www.isi.edu/nsnam/ $\mathrm{ns} /$.

[26] N. Wisitponghpan, F. Bai, P. Mudalige, V. Sadekar, and O. Tonguz, "Routing in sparse vehicular ad hoc wireless networks," IEEE Journal on Selected Areas in Communications, vol. 25, no. 8, pp. 1538-1556, 2007.

[27] Y. H. Ho, A. H. Ho, and K. A. Hua, "Routing protocols for inter-vehicular networks: a comparative study in highmobility and large obstacles environments," Computer Communications, vol. 31, no. 12, pp. 2767-2780, 2008. 

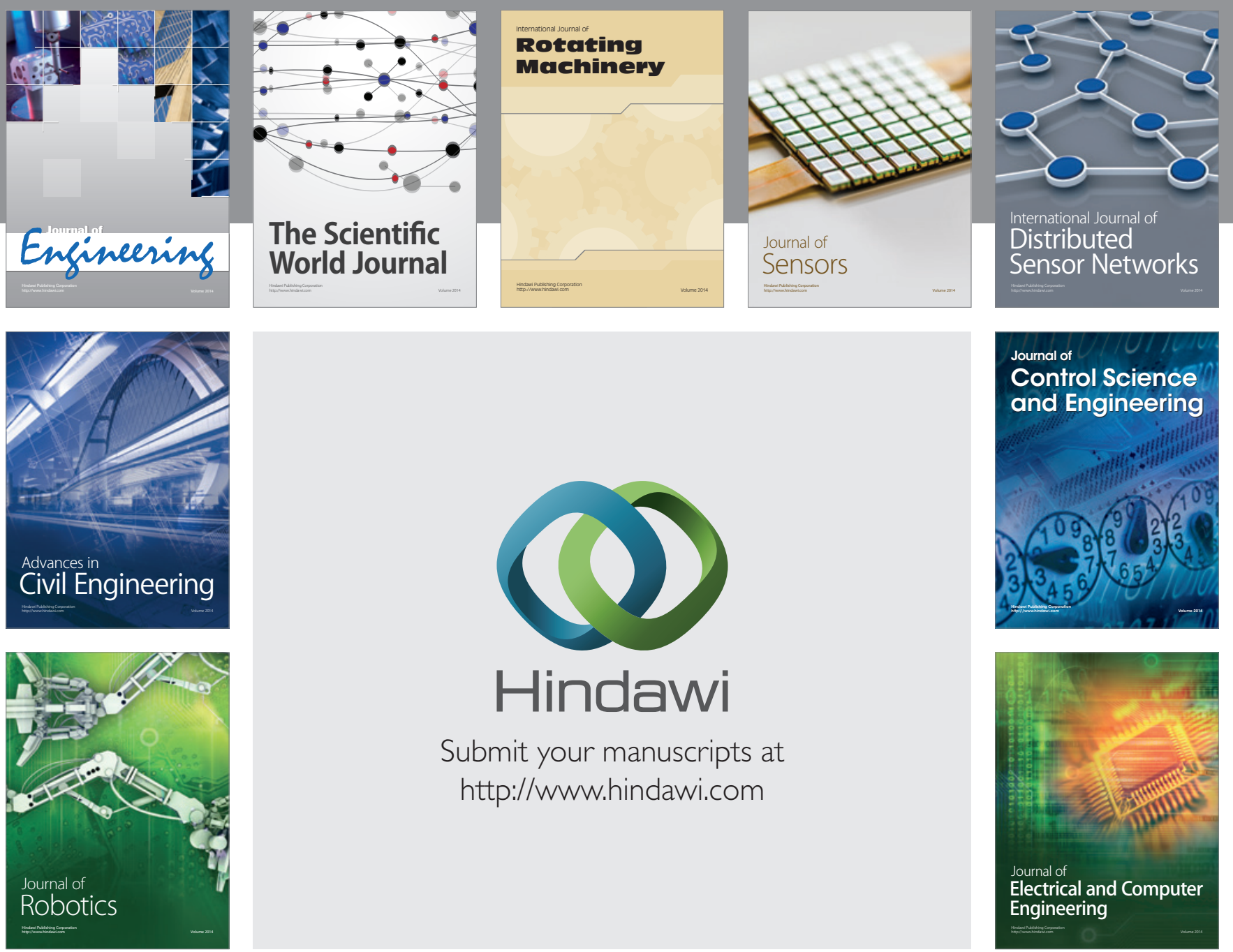

Submit your manuscripts at

http://www.hindawi.com
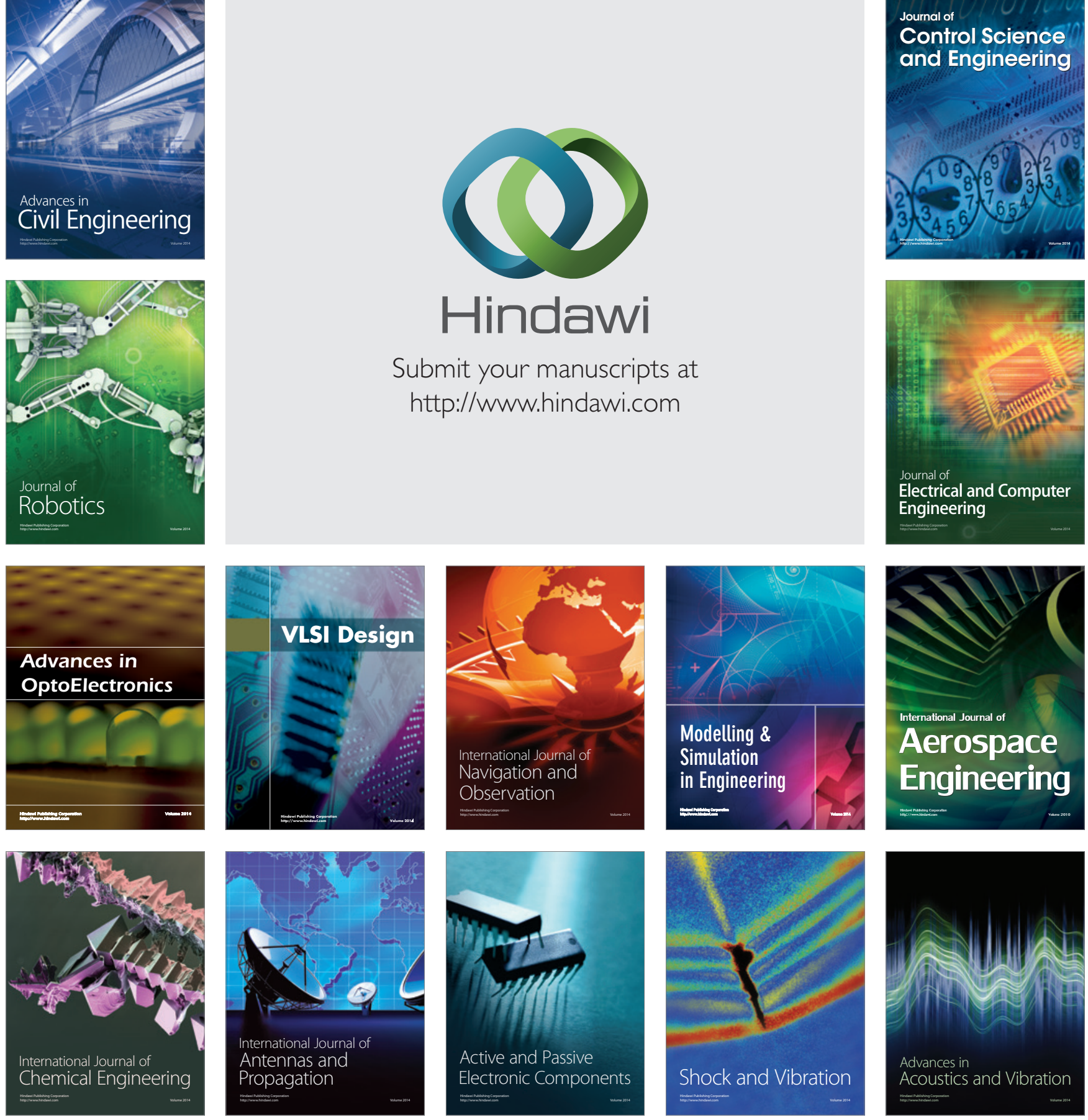